\title{
Eigenvalue Ratio Detection Based On Exact Moments of Smallest and Largest Eigenvalues
}

\author{
Muhammad Zeeshan Shakir*, Wuchen Tang ${ }^{\dagger}$, Anlei Rao*, Muhammad Ali Imran ${ }^{\dagger}$, Mohamed-Slim Alouini* \\ ${ }^{*}$ Division of Physical Sciences and Engineering, KAUST \\ Makkah Province, Thuwal 23955-6900, Kingdom of Saudi Arabia \\ Email: muhammad.shakir@kaust.edu.sa, anlei.rao@kaust.edu.sa, slim.alouini@kaust.edu.sa \\ ${ }^{\dagger}$ Centre for Communication Systems Research (CCSR), University of Surrey \\ Guildford GU1 7XH, Surrey, United Kingdom \\ Email: w.tang@surrey.ac.uk,m.imran@ surrey.ac.uk
}

\begin{abstract}
Detection based on eigenvalues of received signal covariance matrix is currently one of the most effective solution for spectrum sensing problem in cognitive radios. However, the results of these schemes always depend on asymptotic assumptions since the close-formed expression of exact eigenvalues ratio distribution is exceptionally complex to compute in practice. In this paper, non-asymptotic spectrum sensing approach to approximate the extreme eigenvalues is introduced. In this context, the Gaussian approximation approach based on exact analytical moments of extreme eigenvalues is presented. In this approach, the extreme eigenvalues are considered as dependent Gaussian random variables such that the joint probability density function (PDF) is approximated by bivariate Gaussian distribution function for any number of cooperating secondary users and received samples. In this context, the definition of Copula is cited to analyze the extent of the dependency between the extreme eigenvalues. Later, the decision threshold based on the ratio of dependent Gaussian extreme eigenvalues is derived. The performance analysis of our newly proposed approach is compared with the already published asymptotic Tracy-Widom approximation approach.
\end{abstract}

Index Terms-Spectrum sensing; eigenvalue ratio based detection; non-asymptotic Gaussian approximation; Copula.

\section{INTRODUCTION}

In cognitive radio networks, the spectrum resources are available for a secondary user (SU) only when they are not occupied by the primary user (PU), which aims at avoidance of intolerable interference. Thus, the SU should be able to detect the presence of the PU. In this context, high probability of accurate detection in spectrum sensing becomes extremely important in the implementation of cognitive radio networks. Eigenvalue ratio (ER) based detection has been considered as an effective and efficient solution to this problem [1-5]. The key advantage of this detection method lies in the fact that there is no need to obtain a prior information of the PU. The main idea is to decide whether the spectrum resources are being used by a PU or not by exploiting the extreme eigenvalues of the received signal covariance matrix.

Based on the resent results of random matrix theory, the ER detector exploit the ratio between the largest and the smallest eigenvalues of the covariance matrix as analytical statistics and determine the decision threshold. The decision threshold is precalculated, which is determined by the distribution of the test statistics $T_{N}$. However, the exact distribution of the test statistics of the ER detector is generally a mathematically intractable function. Some semi-analytical approaches for the distribution are presented in [5,6] where the computational complexity becomes intractable with the increase in number of SUs $K$ and received samples $N$. The exact expression for this ratio has also been derived in [3], however the distribution can only be evaluated numerically. Also, when $K$ and $N$ are large, the complexity of the exact expression may become computationally cumbersome. As a consequence, a Gaussian approximation is introduced in [7] to derive the analytical distribution of $T_{N}$ such that the decision threshold $\gamma$ can be calculated in a closed-form. Despite the simplicity of the decision threshold, the proposed approximation is only valid under the assumption that the distribution of the largest and the smallest eigenvalues converges to the Tracy-Widom distribution of order two [8]. It has been also shown that such convergence only occurs when $K \rightarrow \infty, N \rightarrow \infty$ and $\frac{K}{N} \rightarrow c \in(0,1)$. However, the resulting cumulative distribution function (CDF) of the Tracy-Widom random variable involve matrix determinants with function entries that are difficult to evaluate when $K$ and $N$ are larger. The derived decision threshold is based on the asymptotic Gaussianity of the extreme eigenvalue distribution obtained by fitting asymptotic moments of Tracy-Widom distribution of order 2 [8]. Moreover, the approximation is under the assumption that the extreme eigenvalues are independent with each other which leads to inaccuracy of considerable extent for moderate to small number of SUs and samples.

The contributions of this paper are as follows: We introduce a simple non-asymptotic spectrum sensing approach based on non-asymptotic Gaussian approximation for any number of collaborating SUs and received samples. The joint probability density function (PDF) of the largest and the smallest eigenvalues are approximated by a bivariate Gaussian distribution function. The proposed approximation captures the non-asymptotic Gaussianity of the extreme eigenvalues based on their exact analytical moments. The definition of Copula is cited to show that the largest and the smallest eigenvalues 
are dependent and their dependency increases with moderate to small values of $K$ and $N$.

The rest of this paper is organized as follows. Section II defines the system model to explain the detection problem of PU; Section III introduces the Gaussian approximation to the extreme eigenvalues. In this section, we recast the Gaussianity of the extreme eigenvalue distribution in the framework of their exact analytical moments. Dependency analysis based on Gaussian Copula between the extreme eigenvalues is also presented; Finally, we calculate an accurate optimal decision threshold for the false alarm probability. Conclusions are presented in IV.

\section{SYSTEM MODEL}

Consider there are $K$ collaborating SUs such that each user collects $N$ samples during the sensing time to detect a PU. The SUs may be considered as a $K$ receive antennas in one secondary terminal or $K$ secondary terminals each with single antenna, or any combinations of these. The collected samples from $K$ collaborating SUs will be forwarded to a fusion center for combined processing. The aim of the SU cognitive phase is to construct and analyze tests associated with the following hypothesis testing problem:

$$
\begin{aligned}
& \mathcal{H}_{0}: \boldsymbol{y}(n)=\boldsymbol{w}(n) \\
& \mathcal{H}_{1}: \boldsymbol{y}(n)=\boldsymbol{h}(n) s(n)+\boldsymbol{w}(n)
\end{aligned}
$$

where $\boldsymbol{y}(n)=\left[y_{1}(n), \cdots, y_{K}(n)\right]^{T}$ is the $K \times 1$ observed complex time series, $\boldsymbol{w}(n)$ represents a $K \times 1$ complex circular Gaussian white noise process with unknown variance $\sigma_{w}^{2}$. In (2), the vector $\boldsymbol{h}(n) \in \mathbb{C}^{K \times 1}$ typically represents the propagation channel between the PU and $K$ collaborating SUs and the signal $s(n)$ denotes a standard scalar i.i.d circular complex Gaussian process w.r.t samples $n=1,2, \cdots, N$ and stands for the source signal to be detected. We stack the observed data into $K \times N$ data matrix $\boldsymbol{Y}$ which may be expressed as

$$
\boldsymbol{Y}=\left(\begin{array}{cccc}
y_{1}(1) & y_{1}(2) & \cdots & y_{1}(N) \\
y_{2}(1) & y_{2}(2) & \cdots & y_{2}(N) \\
\vdots & \vdots & \ddots & \vdots \\
y_{K}(1) & y_{K}(2) & \cdots & y_{K}(N)
\end{array}\right)
$$

The received sampled covariance matrix is $R=Y Y^{H}$ with ordered eigenvalues $\lambda_{1}>\lambda_{2}>\cdots>\lambda_{K}>0$, where $(\cdot)^{H}$ is the Hermitian conjugate operator. These eigenvalues behave differently depending on the presence or absence of the PU. The spectral properties of the covariance matrix is the reason of using the ratio between the largest and the smallest eigenvalues of this matrix as a test statistics to infer the presence or absence of the PU. The test statistics for ER detector is $T_{N}=\lambda_{1} / \lambda_{K}$ and denoting $\gamma$ as the decision threshold employed by the detector such that $T_{N} \underset{\mathcal{H}_{0}}{\stackrel{\mathcal{H}_{1}}{\gtrless}} \gamma$ to decide if the target spectrum resource is occupied or not.

\section{NON-ASYMPtotic GAUSSIAN ApPROXIMATION For ER DETECTOR}

In this section, we calculate the decision threshold for ER detector based on the Gaussian distribution by exchanging its moments with the exact analytical moments of $\lambda_{1}$ and $\lambda_{K}$.

\section{A. Moments of Largest and Smallest Eigenvalues}

Let us denote $\operatorname{det}(\boldsymbol{A}, \boldsymbol{B})$ as the cross-determinant of two matrices $\boldsymbol{A}=\left\{a_{i, j}\right\}_{m \times m}$ and $\boldsymbol{B}=\left\{b_{i, j}\right\}_{n \times n}$ with $m \leq n$ such that

$$
\operatorname{det}(\boldsymbol{A}, \boldsymbol{B})=\sum_{i=1}^{m} \sum_{j=1}^{m}(-1)^{i+j} a_{i, j} B_{i, j}
$$

where $B_{i, j}$ is the minor of matrix $B$ with $i$-th row and $j$-th column deleted. The PDF of the largest $\left(\lambda_{1}\right)$ and the smallest $\left(\lambda_{K}\right)$ eigenvalues are given by [9]

$$
f_{\lambda(\cdot)}(\lambda)=\mathbb{C}_{N, K}^{-1} \operatorname{det}\left(\boldsymbol{A}, \boldsymbol{B}^{(\cdot)}\right)
$$

where the constant $\mathbb{C}_{N, K}=\Gamma_{(K)}(N) \Gamma_{(K)}(K)$ with $\Gamma_{(m)}(n)=\prod_{i=1}^{m}(n-i) !$; the element of $K \times K$ matrix $A$ is $a_{i, j}=e^{-\lambda} \lambda^{N-K+i+j-2}$; the elements of $K \times K$ matrix $\boldsymbol{B}^{(\cdot)}$ for $\lambda_{1}$ and $\lambda_{K}$ are $b_{i, j}=\gamma(N-K+i+j-1, \lambda)$ and $b_{i, j}=\Gamma(N-K+i+j-1, \lambda)$ respectively, with $\gamma(\cdot, \cdot)$ and $\Gamma(\cdot, \cdot)$ as the lower and the upper incomplete gamma functions respectively.

Considering the $p$-th moment of $\lambda_{1}$ and $\lambda_{K}$, we have

$$
\begin{aligned}
\mathbb{E}\left\{\lambda_{(\cdot)}^{p}\right\} & =\mathbb{C}_{N, K}^{-1} \int_{0}^{\infty} \operatorname{det}\left(\boldsymbol{A}_{p}, \boldsymbol{B}^{(\cdot)}\right) d \lambda \\
& =\mathbb{C}_{N, K}^{-1} \sum_{i=1}^{m} \sum_{j=1}^{m}(-1)^{i+j} \int_{0}^{\infty} a_{i, j} B_{i, j} d \lambda
\end{aligned}
$$

where the element of $\boldsymbol{A}_{p}$ are $a_{i, j}=e^{-\lambda} \lambda^{p+N-K+i+j-2}$.

1) Moments of the Smallest Eigenvalue $\left(\lambda_{K}\right)$ : For the smallest eigenvalue $\left(\lambda_{K}\right)$, we have the minors of matrix $\boldsymbol{B}^{(\cdot)}$ as

$$
B_{i, j}=\sum_{\alpha} \operatorname{sgn}(\alpha) \prod_{k=1}^{K-1} \Gamma\left(N-K+L_{\alpha_{k}, k}, \lambda\right),
$$

where $L_{\alpha_{k}, k}$ can be determined by

$$
L_{\alpha_{k}, k}= \begin{cases}k+\alpha_{k}-1 & \text { if } \alpha_{k}<i \text { and } k<j \\ k+\alpha_{k}+1 & \text { if } \alpha_{k} \geq i \text { and } k \geq j \\ k+\alpha_{k} & \text { otherwise }\end{cases}
$$

Note that $\Gamma(n, \lambda)=e^{-\lambda}(n-1) ! \sum_{l=0}^{n-1} \frac{\lambda^{l}}{l !}$, then $B_{i, j}$ may be expressed as

$$
\begin{aligned}
B_{i, j}= & \sum_{\alpha} \operatorname{sgn}(\alpha) \mathrm{e}^{-(K-1) \lambda} \prod_{k=1}^{K-1}\left(N-K+L_{\alpha_{k}, k}-1\right) ! \\
& \times\left(\sum_{l_{1 \sim(K-1)}}^{L_{1 \sim(K-1)}} \frac{\lambda^{\sum l_{1}^{(K-1)}}}{l_{1}^{(K-1)} !}\right)
\end{aligned}
$$

where

$$
\sum_{l_{1 \sim n}}^{L_{1 \sim n}}=\sum_{l_{1}=0}^{\left(N-K+L_{\alpha_{1}, 1}-1\right)} \sum_{l_{2}=0}^{\left(N-K+L_{\alpha_{2}, 2}-1\right)} \cdots \sum_{l_{n}=0}^{\left(N-K+L_{\alpha_{n}, n}-1\right)} ;
$$




$$
\begin{aligned}
\mathbb{E}\left\{\lambda_{K}^{p}\right\} & =\mathbb{C}_{N, K}^{-1} \sum_{i, j=1}^{K}(-1)^{i+j} \sum_{\alpha} \operatorname{sgn}(\alpha) \prod_{k=1}^{K-1}\left(N-K+L_{\alpha_{k}, k}-1\right) !\left(\sum_{l_{1 \sim K-1}}^{L_{1 \sim K-1}} \frac{1}{l_{1}^{(K-1)} !} \int_{0}^{\infty} e^{-K \lambda} \lambda^{\left(\sum l_{1}^{K-1}+N-K+i+j+p-2\right)} d \lambda\right) \\
& =\mathbb{C}_{N, K}^{-1} \sum_{i, j=1}^{K}(-1)^{i+j} \sum_{\alpha} \operatorname{sgn}(\alpha) \prod_{k=1}^{K-1}\left(N-K+L_{\alpha_{k}, k}-1\right) !\left(\sum_{l_{1 \sim K-1}}^{L_{1 \sim K-1}} \frac{\Gamma\left(\sum l_{1}^{K-1}+N-K+i+j+p-1\right)}{l_{1}^{(K-1)} ! K l^{K-1}+N-K+i+j+p-1}\right) \\
\mathbb{E}\left\{\lambda_{1}^{p}\right\} & =\mathbb{C}_{N, K}^{-1} \sum_{i, j=1}^{K}(-1)^{i+j} \sum_{\alpha} \operatorname{sgn}(\alpha) \prod_{k=1}^{K-1}\left(N-K+L_{\alpha_{k}, k}-1\right) !\left(\sum_{\mathbb{S}}(-1)^{|\mathbb{S}|} \frac{\Gamma\left(\sum \mathbb{S}+N-K+i+j+p-1\right)}{\prod \mathbb{S} !(|\mathbb{S}|+1)^{\sum \mathbb{S}+N-K+i+j+p-1}}\right)
\end{aligned}
$$

$\sum l_{1}^{n}=\left(l_{1}+l_{2}+\cdots+l_{n}\right)$ and $l_{1}^{n} !=l_{1} ! l_{2} ! \cdots l_{n} !$

By substituting (10) into (7), we have (11) which is showing at top of the page. The $p$-th moment of the smallest eigenvalue $\left(\lambda_{K}\right)$ can be calculated using (12).

2) Moments of the Largest Eigenvalue $\left(\lambda_{1}\right)$ : Similarly, for the largest eigenvalue $\left(\lambda_{1}\right)$, we have

$$
B_{i, j}=\sum_{\alpha} \operatorname{sgn}(\alpha) \prod_{k=1}^{K-1} \gamma\left(N-K+L_{\alpha_{k}, k}, \lambda\right),
$$

where $L_{\alpha_{k}, k}$ is determined by (9). Note that $\gamma(n, \lambda)=(n-1) !\left(1-e^{-\lambda} \sum_{l=0}^{n-1} \frac{\lambda^{l}}{l !}\right)$, we may express $B_{i, j}$ as

$$
\begin{aligned}
B_{i, j}= & \sum_{\alpha} \operatorname{sgn}(\alpha) \prod_{k=1}^{K-1}\left(N-K+L_{\alpha_{k}, k}-1\right) ! \\
& \times \prod_{k=1}^{K-1}\left(1-e^{-\lambda} \sum_{l_{k}=0}^{N-K+L_{\alpha_{k}, k}-1} \frac{\lambda^{l_{k}}}{l_{k} !}\right) \\
= & \sum_{\alpha} \operatorname{sgn}(\alpha) \prod_{k=1}^{K-1}\left(N-K+L_{\alpha_{k}, k}-1\right) ! \\
& \times\left(\sum_{\mathbb{S}}\left(-e^{-\lambda}\right)^{|\mathbb{S}|} \frac{\lambda^{\sum \mathbb{S}}}{\prod \mathbb{S} !}\right),
\end{aligned}
$$

where $\mathbb{S}$ is any subset of the set $\left\{l_{1}, l_{2}, \cdots, l_{K-1}\right\}$ with $l_{k}$ from 0 to $N-K+L_{\alpha_{k}, k}-1, \sum_{\mathbb{S}}$ is the sum over all the elements in $\mathbb{S},|\mathbb{S}|$ is the cardinality of subset $\mathbb{S}, \sum \mathbb{S}$ is the sum of all the elements in the subset $\mathbb{S}$, and $\prod \mathbb{S}$ ! is the product of the factoring of each element in $\mathbb{S}$. For example, if $\mathbb{S}=$ $\left\{l_{i_{1}}, l_{i_{2}}, \cdots, l_{i_{k}}\right\}$, then we have $|\mathbb{S}|=k, \sum \mathbb{S}=l_{i_{1}}+l_{i_{2}}+$ $\cdots+l_{i_{k}}, \prod \mathbb{S} !=l_{i_{1}} ! l_{i_{2}} ! \cdots l_{i_{k}} !$ and

$$
\sum_{\mathbb{S}}=\sum_{l_{i_{1}}=0}^{\left(N-K+L_{\alpha_{i_{1}, i_{1}}}-1\right)} \sum_{l_{i_{2}}=0}^{\left(N-K+L_{\alpha_{i_{2}, i_{2}}}-1\right)} \cdots \sum_{l_{i_{k}}=0}^{\left(N-K+L_{\alpha_{i_{k}}, i_{k}}-1\right)} \text {; }
$$

For the special case, when $\mathbb{S}$ is empty, we have $|\mathbb{S}|=0, \sum \mathbb{S}=$ 0 and $\prod \mathbb{S} !=1$. With $B_{i, j}$ above, the $p$-th moment of the largest eigenvalue $\left(\lambda_{1}\right)$ can be calculated as given in (16).

Choose $p=1$ and $p=2$ to calculate the first and the second moments respectively of $\lambda_{K}$ and $\lambda_{1}$. The numerical values of mean and variance of the extreme eigenvalues are
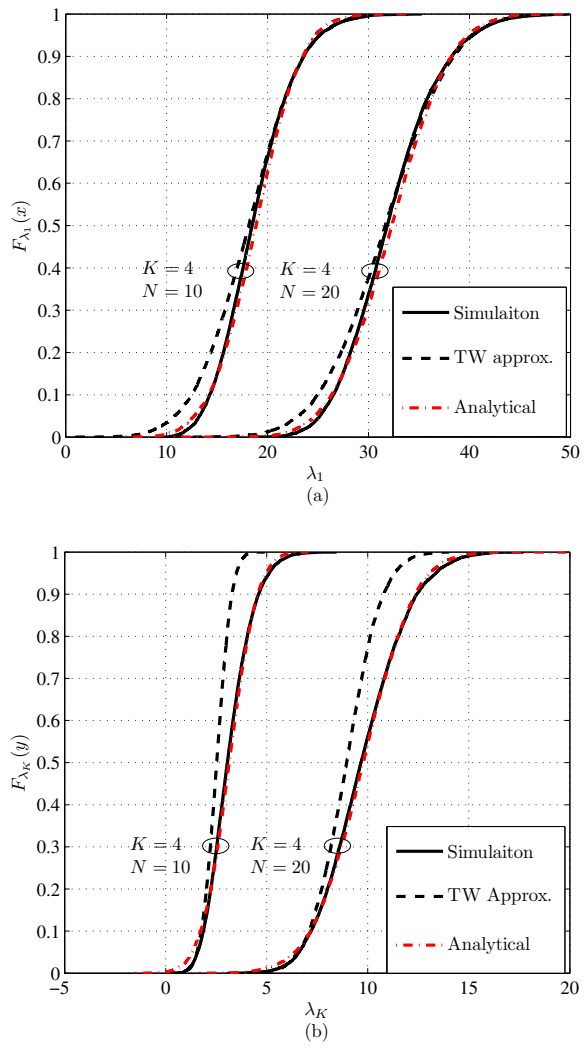

Fig. 1: CDFs of :(a) the largest eigenvalue; (b) the smallest eigenvalue.

shown in Table I. The CDFs of the largest and the smallest eigenvalues for some selected values of $K$ and $N$ are shown in Fig. 1(a) and Fig. 1(b) respectively. The figures illustrate that the proposed non-asymptotic Gaussian approximation based on exact moments of extreme eigenvalues outperforms the approximation based on the moments of the Tracy-Widom distribution. Moreover, our results are in perfect agreement with the empirical results.

\section{B. Joint PDF and Copula of Extreme Eigenvalues}

We employ the exact analytical moments of extreme eigenvalues to recast the bivariate Gaussian distribu- 
Table I: Numerical results of largest and smallest eigenvalues

\begin{tabular}{|c|c|c|c|c|c|c|c|c|c|c|c|c|}
\hline \multirow{3}{*}{$(K, N)$} & \multicolumn{6}{|c|}{$\lambda_{1}$} & \multicolumn{6}{|c|}{$\overline{\lambda_{K}}$} \\
\hline & \multicolumn{2}{|c|}{ Simulated } & \multicolumn{2}{|c|}{ Tracy Widom Approx. } & \multicolumn{2}{|c|}{ Analytical } & \multicolumn{2}{|c|}{ Simulated } & \multicolumn{2}{|c|}{ Tracy Widom Approx. } & \multicolumn{2}{|c|}{ Analytical } \\
\hline & mean & variance & mean & $\begin{array}{l}\text { variance } \\
\text { varis }\end{array}$ & mean & variance & mean & variance & mean & variance & mean & variance \\
\hline$(2,4)$ & 6.1857 & 5.4267 & 5.2184 & 10.7467 & 6.1875 & 5.4023 & 1.8161 & 1.0328 & 0.9570 & 0.0977 & 1.8125 & 1.0273 \\
\hline$(2,10)$ & 13.5144 & 11.0854 & 12.7763 & 17.2958 & 13.5239 & 11.1058 & 6.4803 & 4.0686 & 5.3194 & 1.3284 & 6.4761 & 4.0579 \\
\hline$(4,20)$ & 32.1538 & 21.1422 & 31.5975 & 27.4554 & 32.1652 & 21.1160 & 9.8012 & 4.2497 & 8.9635 & 2.1088 & 9.8047 & 4.2215 \\
\hline$(4,50)$ & 68.8106 & 42.6479 & 68.4290 & 49.7672 & 68.7793 & 42.4981 & 33.1711 & 15.7900 & 32.0965 & 10.5549 & 33.1032 & 15.8013 \\
\hline
\end{tabular}

tion function as a joint PDF of extreme eigenvalues. Let $\left(\lambda_{1} \sim \mathcal{N}\left(\mu_{\lambda_{1}}, \sigma_{\lambda_{1}}^{2}\right), \lambda_{K} \sim \mathcal{N}\left(\mu_{\lambda_{K}}, \sigma_{\lambda_{K}}^{2}\right)\right)$ be a bivariate Gaussian random variables with

$$
\boldsymbol{\mu}=\left(\begin{array}{c}
\mu_{\lambda_{1}} \\
\mu_{\lambda_{K}}
\end{array}\right) \text { and } \boldsymbol{\Sigma}=\left(\begin{array}{cc}
\sigma_{\lambda_{1}}^{2} & \rho \sigma_{\lambda_{1}} \sigma_{\lambda_{K}} \\
\rho \sigma_{\lambda_{1}} \sigma_{\lambda_{K}} & \sigma_{\lambda_{K}}^{2}
\end{array}\right)
$$

are the mean and the covariance of bivariate Gaussian distribution function respectively, and where $\rho$ is the correlation coefficient between $\lambda_{K}$ and $\lambda_{1}$. If $f_{\lambda_{1}, \lambda_{K}}(x, y)$ is the joint PDF of the extreme eigenvalues then by exchanging the Gaussian moments with the exact analytical moments of extreme eigenvalues, the joint PDF can be approximated as

$f_{\lambda_{1}, \lambda_{K}}(x, y)=\frac{1}{2 \pi \sigma_{\lambda_{1}} \sigma_{\lambda_{K}}\left(1-\rho^{2}\right)^{0.5}} \exp \left(-\frac{z}{2(1-\rho)^{2}}\right)$

where

$z \equiv \frac{\left(x-\mu_{\lambda_{1}}\right)^{2}}{\sigma_{\lambda_{1}}^{2}}-\frac{2 \rho\left(x-\mu_{\lambda_{1}}\right)\left(y-\mu_{\lambda_{K}}\right)}{\sigma_{\lambda_{1}} \sigma_{\lambda_{K}}}+\frac{\left(y-\mu_{\lambda_{K}}\right)^{2}}{\sigma_{\lambda_{K}}^{2}}$

Now, we analyze the dependency between the random variables $\left(\lambda_{1}, \lambda_{K}\right)$ to approximate $\rho$ by plotting their Copula. A Copula is a multivariate distribution function with known marginal cumulative distribution functions (CDFs) [10]. More specifically, a bivariate joint distribution function $F_{\lambda_{1}, \lambda_{K}}(x, y)=\operatorname{Pr}\left\{\lambda_{1} \leq x, \lambda_{K} \leq y\right\}$ of two random variables $\lambda_{1}$ and $\lambda_{K}$, may be represented by a Copula $C$ as a function of their marginal CDFs $F_{\lambda_{1}}(x)=\operatorname{Pr}\left\{\lambda_{1} \leq x\right\}$ and $F_{\lambda_{K}}(y)=\operatorname{Pr}\left\{\lambda_{K} \leq y\right\}$ and therefore may be expressed as [10]

$$
F_{\lambda_{1}, \lambda_{K}}(x, y)=C\left(F_{\lambda_{1}}(x), F_{\lambda_{K}}(y)\right) \triangleq C(u, v)
$$

where $u=F_{\lambda_{1}}(x)$ and $v=F_{\lambda_{K}}(y) ; C(u, v)$ is the associated Copula distribution function. Thus, we have

$$
C(u, v)=F_{\lambda_{1}, \lambda_{K}}\left(F_{\lambda_{1}}^{-1}(u), F_{\lambda_{K}}^{-1}(v)\right)
$$

By exploiting the chain rule, the corresponding joint PDF $f_{\lambda_{1}, \lambda_{K}}(x, y)$ may be decomposed as

$$
\begin{gathered}
f_{\lambda_{1}, \lambda_{K}}(x, y)=\frac{\partial^{2} F_{\lambda_{1}, \lambda_{K}}(x, y)}{\partial x \partial y}=\frac{\partial^{2} C\left(F_{\lambda_{1}}(x), F_{\lambda_{K}}(y)\right)}{\partial x \partial y} \\
=\frac{\partial^{2} C(u, v)}{\partial u \partial v} \frac{\partial F_{\lambda_{1}}(x)}{\partial x} \frac{\partial F_{\lambda_{K}}(y)}{\partial y} \triangleq c(u, v) f_{\lambda_{1}}(x) f_{\lambda_{K}}(y)
\end{gathered}
$$

It is obvious that the joint PDF is the product of the marginal PDFs $f_{\lambda_{1}}(x)$ and $f_{\lambda_{K}}(y)$ and Copula density function $c(u, v)$. The definition of Copula identifies a strong relationship which provides link between the marginal PDFs/CDFs and the respective joint PDF/CDF. Also, $c(u, v)=1$, for independent random variables [10].
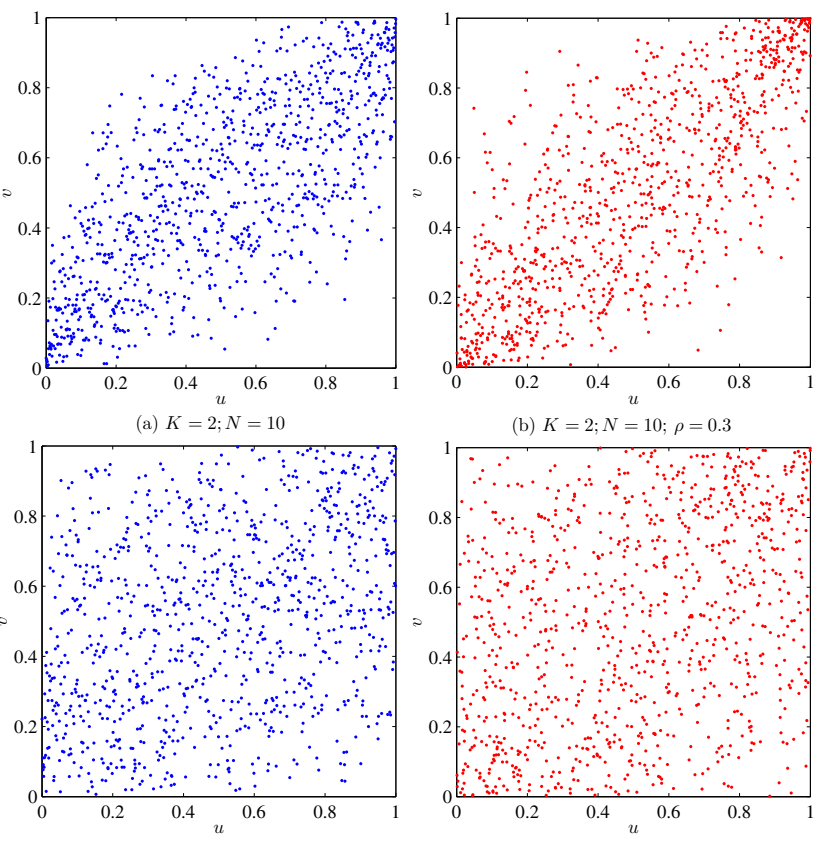

(c) $K=4 ; N=50$

(d) $K=4 ; N=50 ; \rho=0.1$

Fig. 2: Plots of Copula between random variables $\lambda_{1}$ and $\lambda_{K}$ based on empirical (blue scatter plot) and Gaussian (red scatter plot) marginal distribution functions.

Let $\Phi_{\lambda_{1}}(x)$ and $\Phi_{\lambda_{K}}(y)$ are the marginal distribution functions of the approximated Gaussian random variables $\lambda_{1}$ and $\lambda_{K}$ respectively. Using the statistical values of $\mu_{\lambda_{1}}, \mu_{\lambda_{K}}, \sigma_{\lambda_{1}}^{2}$, $\sigma_{\lambda_{K}}^{2}$, the Copula distribution of (17) is given by [10]

$$
C(u, v)=\int_{-\infty}^{\Phi_{\lambda_{1}}^{-1}(u)} \int_{-\infty}^{\Phi_{\lambda_{K}}^{-1}(v)} f_{\lambda_{1}, \lambda_{K}}(x, y) d x d y
$$

where $\Phi_{(\cdot)}^{-1}(\cdot)$ is the inverse of the standard univariate Gaussian distribution function.

The plots of Copula between $\lambda_{1}$ and $\lambda_{K}$ based on the empirical and Gaussian marginal distribution functions are showing in Fig. 2(a) and Fig. 2(b) respectively for $(K, N)=(2,10)$. It can be seen that the structure of Copula based on the empirical distribution functions appears same as the Copula structure based on the Gaussian distribution functions for $\rho=0.3$. Similar kind of observation can be made from Fig. 2(c) and Fig. 2(d) when $(K, N)=(4,50)$ and correlation reduces to $\rho=0.1$. It can also be noticed that the Copula structure is distinctive for small value of $K$ and $N$, i.e. the extreme eigenvalues are dependent. However, with the increase in number of $K$ and $N$, the dependency between $\lambda_{1}$ and $\lambda_{K}$ decreases. The 


$$
\gamma=\frac{\mu_{\lambda_{1}} \mu_{\lambda_{K}}-\tau^{2} \rho \sigma_{\lambda_{1}} \sigma_{\lambda_{K}}}{\mu_{\lambda_{K}}^{2}-\tau^{2} \sigma_{\lambda_{K}}^{2}}+\frac{\tau \sqrt{\mu_{\lambda_{1}}^{2} \sigma_{\lambda_{K}}^{2}+\mu_{\lambda_{K}}^{2} \sigma_{\lambda_{1}}^{2}+\left(\rho^{2}-1\right) \tau^{2} \sigma_{\lambda_{1}}^{2} \sigma_{\lambda_{K}}^{2}-2 \mu_{\lambda_{1}} \mu_{\lambda_{K}} \rho \sigma_{\lambda_{1}} \sigma_{\lambda_{K}}}}{\mu_{\lambda_{K}}^{2}-\tau^{2} \sigma_{\lambda_{K}}^{2}}
$$

same is shown in Fig. 2. Therefore, the dependency between $\lambda_{1}$ and $\lambda_{K}$ can not be ignored if accurate spectrum sensing is required. However, some analytical approaches may be applied to calculate the exact value of $\rho$.

\section{Calculation of Decision Threshold}

We have already shown that the joint PDF of $\left(\lambda_{1}, \lambda_{K}\right)$ can be well approximated by a bivariate Gaussian distribution function knowing their exact moments and $\rho$. If the joint PDF of $\left(\lambda_{1}, \lambda_{K}\right) \sim \mathcal{N}(\boldsymbol{\mu}, \boldsymbol{\Sigma})$ is given as (17), then the CDF of the ratio of two dependent Gaussian random variables may be expressed as [11]

$$
F(\gamma)=\Phi\left\{\frac{\mu_{\lambda_{K}} \gamma-\mu_{\lambda_{1}}}{\sigma_{\lambda_{1}} \sigma_{\lambda_{K}} a(\gamma)}\right\}
$$

where $a(\gamma)=\left(\frac{\gamma^{2}}{\sigma_{\lambda_{1}}^{2}}-\frac{2 \rho \gamma}{\sigma_{\lambda_{1}} \sigma_{\lambda_{K}}}+\frac{1}{\sigma_{\lambda_{K}}^{2}}\right)^{0.5}$ and $\Phi($.$) is the$ $\mathrm{CDF}$ of a standard Gaussian random variable. Using (22), we may calculate an accurate decision threshold analytically in closed form based on exact moments. For a given target false alarm probability $\left(P_{f a}\right)$ and fixed $\rho$, the decision threshold $\gamma$ is obtained by solving

$$
P_{f a}=1-F(\gamma)
$$

The solution is shown at the top of the page as (24), where $\tau=\Phi^{-1}\left(1-P_{f a}\right)$.

In Fig. 3, we plot the decision threshold $\gamma$ as a function of $P_{f a}$ for small and moderate number of $K$ and $N$ to compare the Gaussianity of $\lambda_{1}$ and $\lambda_{K}$ based on exact moments with their Gaussianity based on the moments of Tracy-Widom distribution. It can be seen that the proposed non-asymptotic Gaussian approximation performs extremely well in comparison with the Tracy-Widom approximation approach and our results match the empirical results. The proposed approximation is equivalently good for any number of $K$ and $N$, however the results are significantly accurate for reasonably moderate to small number of $K$ and $N$.

\section{CONCLUSION}

In this paper, a non-asymptotic Gaussian approximation based on the exact analytical moments of the extreme eigenvalues has been employed to approximate the decision threshold of ER detector. Copula plots show that the extreme eigenvalues are dependent and their dependency should not be ignored specially for moderate to small number of $N$ and $K$. The accuracy of decision threshold based on Gaussian approximation has been evaluated in comparison with decision threshold calculated using Tracy-Widom approximation approach. It has been shown that our proposed approach is accurate enough for any number of $N$ and $K$.
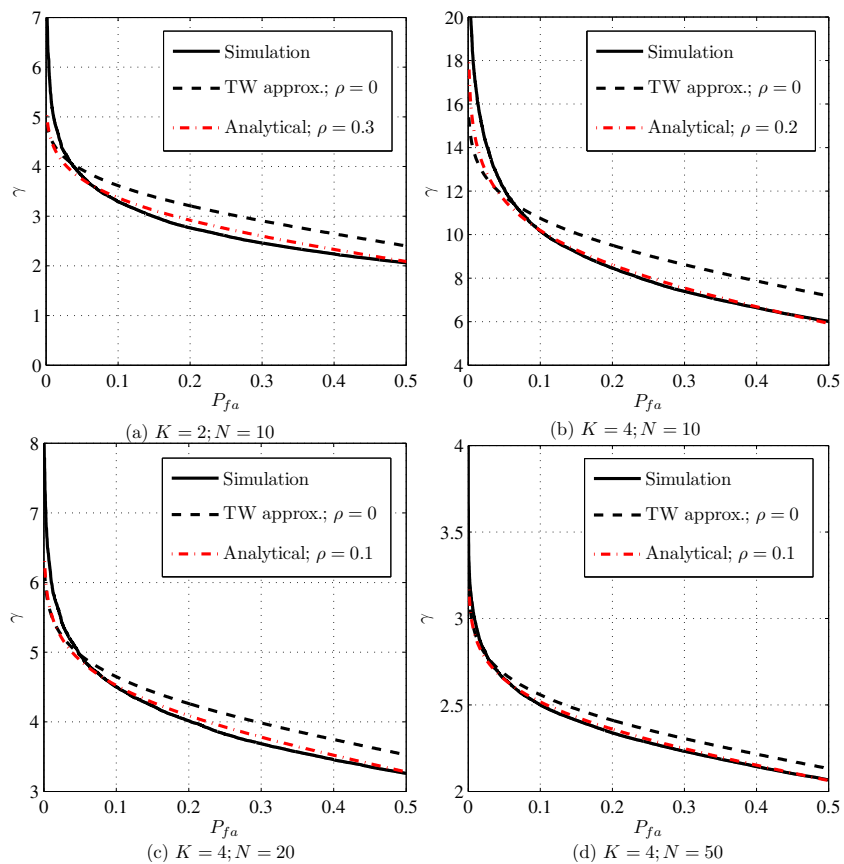

Fig. 3: Decision threshold vs. $P_{f a}$ for selected values of $K$ and $N$.

\section{REFERENCES}

[1] Y. Zeng and Y. C. Liang, "Eigenvalue based spectrum sensing algorithms for cognitive radios," in IEEE Trans. Communs., vol. 57, no. 6, pp. 1784-1793, Jun. 2009.

[2] F. Penna, R. Garello and M. A. Spirito, "Cooperative spectrum sensing based on the limiting eigenvalue ratio distribution in Wishart matrices," in IEEE Communs. Letters, vol. 13, no. 7, pp. 507-509, Jul. 2009.

[3] F. Penna, R. Garello, D. Figlioli and M. A. Spirito, "Exact nonasymptotic threshold for eigenvalue-based spectrum sensing," in Proc. ICST Conf. Cognitive Radio Oriented Wireless Networks and Communs., CrownCom'2009, Hannover, Germany, Jun. 2009.

[4] Y. Zeng, Y.-C. Liang, A. T. Hoang, and R. Zhang, "A review on spectrum sensing for cognitive radio: challenges and solutions," in EURASIP Jour. Advances in Signal Processing, vol. 2010, Article ID 381465, 2010.

[5] Y. Zeng and Y. Liang, "Maximum-minimum eigenvalue detection for cognitive radio," in IEEE Intl. Conf. Personal, Indoor and Mobile Radio Communs., PIMRC'2007, Athens, Greece, Sep. 2007.

[6] L. S. Cardoso, M. Debbah, P. Bianchi and J. Najim, "Cooperative spectrum sensing using random matrix theory," in Proc. Intl. Symp. Wireless Pervasive Computing, ISWPC'2008, pp. 334-338, Santorini, Greece, Jul. 2008.

[7] L. Wei and O. Tirkkonen, "Spectrum sensing with Gaussian approximated eigenvalue ratio based detection," in Proc. IEEE Intl. Symp. Wireless Commun. Systems, ISWCS'2010, pp. 961-965, York, UK, Sep. 2010.

[8] C. Tracy and H. Widom, "On orthogonal and symplectic matrix ensembles," in Springer Jour. Communs. in Mathematical Physics., vol. 177, no. 3, pp. 727-754, 1996.

[9] A. Zanella, M. Chiani and M. Z. Win, "On the marginal distribution of the eigenvalues of Wishart matrices," in IEEE Trans. Communs., vol. 57, no. 4, pp. 1050-1060, Apr. 2009.

[10] T. S. Durrani and X. Zeng, "Copula for bivariate probability distribution," in IET Electronics Letters, vol. 43, no. 4, pp. 248-249, Feb. 2007.

[11] D. V. Hinkley, "On the ratio of two correlated normal random variables," in Oxford Jour. Biometrika, vol. 56, no. 3, pp. 635-639, Dec. 1969. 